\title{
Intrathecal synthesis of oligoclonal IgM against myelin lipids predicts an aggressive disease course in MS
}

\author{
Luisa M. Villar, ${ }^{1}$ María C. Sádaba, ${ }^{1}$ Ernesto Roldán, ${ }^{1}$ Jaime Masjuan, ${ }^{1}$ Pedro González-Porqué, ${ }^{1}$ \\ Noelia Villarrubia, ${ }^{1}$ Mercedes Espiño, ${ }^{1}$ José A. García-Trujillo, ${ }^{1}$ Alfredo Bootello, ${ }^{1}$ \\ José C. Álvarez-Cermeño1,2
}

1Departments of Immunology and Neurology, Hospital Ramón y Cajal, Madrid, Spain. ${ }^{2}$ Department of Medicine, University of Alcalá de Henares, Madrid, Spain.

\begin{abstract}
Oligoclonal IgM bands restricted to cerebrospinal fluid are an unfavorable prognostic marker in MS, the most common demyelinating disease of the CNS. We have attempted to identify the B cell subpopulation responsible for oligoclonal IgM secretion and the specificity of these bands. In addition, we explored the relationship between specificity and disease evolution. Intrathecal B cell subpopulations present in 29 MS patients with oligoclonal IgM bands and 52 without them were analyzed. A considerable increase in CD5 ${ }^{+}$ B lymphocytes was found in patients with oligoclonal IgM bands. These cells mostly secrete IgM antibodies recognizing nonproteic molecules. We also studied whether oligoclonal IgM bands present in cerebrospinal fluid of $53 \mathrm{MS}$ patients were directed against myelin lipids. This was the case in most patients, with phosphatidylcholine being the most frequently recognized lipid. Disease course of 15 patients with oligoclonal IgM against myelin lipids and 33 patients lacking them was followed. Patients with anti-lipid IgM suffered a second relapse earlier, had more relapses, and showed increased disability compared with those without anti-lipid IgM. The presence of intrathecal anti-myelin lipid IgM antibodies is therefore a very accurate predictor of aggressive evolution in MS.
\end{abstract}

\section{Introduction}

MS is a demyelinating disease of the CNS of unknown etiology. The clinical course begins with a pattern of relapse and remission that continues for variable lengths of time. This may be followed by a second phase that is characterized by a continuous progression of disability. In about $20 \%$ of patients, disability is progressive from the onset; in those cases, the disease is termed "primary progressive MS."

It has been postulated that MS is an autoimmune disease, and considerable efforts have been made to find a particular antigen associated with its physiopathology (1-4). The presence of T cells, macrophages, and antibodies in MS demyelinating plaques has been established (5). It has been postulated that T cells and macrophages play an important role in the inflammatory response observed in MS and that antibodies and complement participate in initial plaque development $(6,7)$. Increasing evidence indicates that IgM antibodies are also involved in this process. In this disease, local CNS (intrathecal) synthesis of IgM, but not of IgG or IgA, correlates with intrathecal synthesis of complement component C3 (8) and with the cerebrospinal fluid (CSF) concentration of myelin basic protein, an index of demyelination (9).

Oligoclonal band detection is the best method for investigating the presence of intrathecal IgM synthesis, which is demonstrated by the presence of oligoclonal IgM bands (OCMBs) restricted to CSF (10). With this method, intrathecal IgM syn-

Nonstandard abbreviations used: CSF, cerebrospinal fluid; EDSS, expanded standard disability status scale; IEF, isoelectric focusing; LS-OCMB, lipid-specific OCMB; $\mathrm{M}+$, with OCMBs; $\mathrm{M}$-, without OCMBs; OCMB, oligoclonal IgM band; PC, phosphatidylcholine.

Conflict of interest: The authors have declared that no conflict of interest exists.

Citation for this article: J. Clin. Invest. 115:187-194 (2005)

doi:10.1172/JCI200522833 thesis has been detected in a subgroup of MS patients $(11,12)$. These IgM bands usually persist in MS patients throughout the course of the disease $(13,14)$ and are an unfavorable prognostic marker (14). This was not unexpected, since IgM is the most efficient immunoglobulin for complement fixation, and it has been demonstrated that complement causes demyelination associated with axonal injury $(15,16)$. In addition, IgM colocalizes with complement in the demyelinating lesions of patients with Devic neuromyelitis optica, a disorder that has been considered to be a form of MS and is characterized by aggressive demyelination (17).

Regarding the nature of intrathecal IgM synthesis in MS, its persistence indicates that it is not a primary immune response, yet it is a persistent one. Persistent IgM responses are usually produced by $\mathrm{CD}^{+} \mathrm{B}$ lymphocytes, a subset of $\mathrm{B}$ cells responsible for the secretion of the so-called natural antibodies, which are usually of IgM isotype and directed against nonproteic antigens $(18,19)$. Studies have reported a higher percentage of $\mathrm{CD}^{+} \mathrm{B}$ lymphocytes in CSF of patients with MS compared with other neurological diseases $(20,21)$. This increase was mainly found in patients with aggressive forms of the disease (21). Taking these data into account, the first purpose of this study was to identify the B cell subpopulation responsible for intrathecal IgM secretion in MS.

CNS myelin is rich in nonproteic molecules (22) and shows little protein content (23). Moreover, the presence of antibodies against myelin lipids has been reported in other demyelinating diseases of the peripheral nervous system $(24,25)$. For these reasons, our second purpose was to study whether the oligoclonal IgM present in CSF of a group of MS patients is directed against myelin lipids and to explore the possible correlation between these antibodies and disease course. 

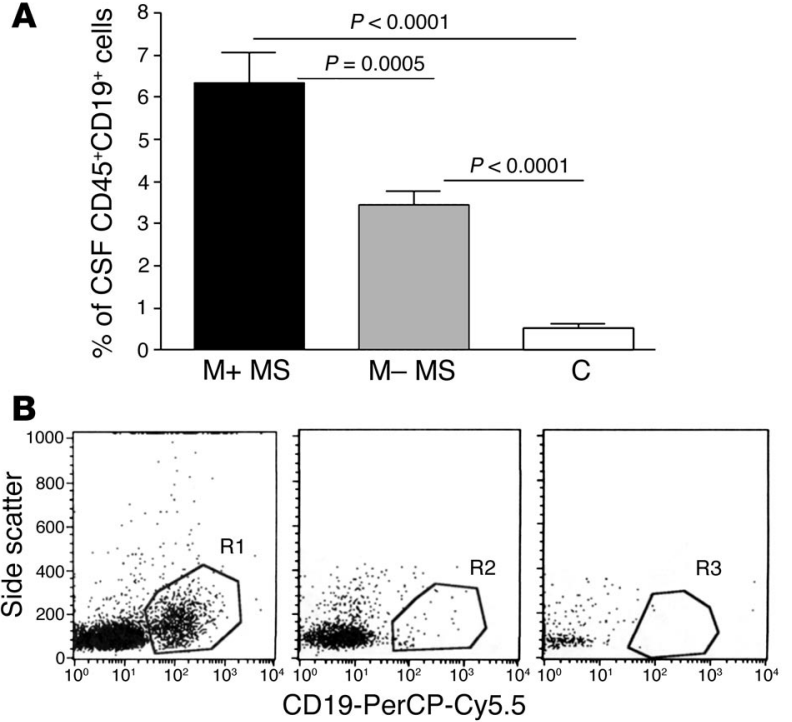

Figure 1

Study of B cells in CSF of MS patients with and without OCMBs. (A) Both $\mathrm{M}+\mathrm{MS}$ and $\mathrm{M}-\mathrm{MS}$ OCMBs showed a higher percentage of CD19+ cells in CSF when compared with the control group $(P<0.0001)$. In addition, M+ MS patients show a higher percentage of $\mathrm{CD} 19^{+}$cells when compared with $\mathrm{M}-\mathrm{MS}$ patients $(P=0.0005)$. (B) Representative dot plots showing CD19+ cells present in CSF of an M+ MS patient (left), an M- MS patient (center), and a patient from the control group (right). CD19-PerCP-Cy5.5, CD19-peridinin-chlorophyll-protein-Cy-Chrome 5.5.

\section{Results}

Immunophenotypic characteristics of B cell subpopulations from CSF of MS patients and controls. The percentage of total B lymphocytes $\left(\mathrm{CD} 19^{+}\right.$cells) present in CSF was analyzed in $29 \mathrm{MS}$ patients with OCMBs (M+ MS patients), $52 \mathrm{MS}$ patients without OCMBs (M- MS patients), and 21 controls (Figure 1A). We found that both $\mathrm{M}+(6.33 \% \pm 0.73 \%$, mean $\pm \mathrm{SE})$ and $\mathrm{M}-(3.46 \% \pm 0.30 \%)$ groups had increased percentages of B lymphocytes compared with the control group $(0.37 \% \pm 0.10 \%)(P<0.0001)$. In addition, differences were also found in $\mathrm{B}$ cell percentages between $\mathrm{M}+$ and M-patients $(P=0.0005)$. Representative dot plots showing the CSF B lymphocytes from an M+ MS patient, an M- MS patient, and a control are shown in Figure 1B.

To study whether the differences in B cell percentages found between $\mathrm{M}+$ and $\mathrm{M}$ - patients could be ascribed to a specific $\mathrm{B}$ cell subpopulation, we analyzed CSF CD5-positive and -negative $\mathrm{B}$ cell subsets (Figure 2). Considerable differences were found in the $\mathrm{CD}^{+}$subset between $\mathrm{M}+(3.94 \% \pm 0.73 \%)$ and M- MS $(1.31 \% \pm 0.17 \%)$ patients $(P<0.0001)$. No differences were found in the $\mathrm{CD}^{-}$subset between $\mathrm{M}+(2.41 \% \pm 0.35 \%)$ and M- $(2.17 \% \pm 0.21 \%)$ MS patients, although they were increased when compared with the control group, where practically no $\mathrm{B}$ cells were found in CSF. Thus, the CSF CD $19^{+} \mathrm{CD}^{-}$subpopulation is increased in both $\mathrm{M}+$ and $\mathrm{M}-$ patients when compared with the control group, and the $\mathrm{CSF} \mathrm{CD} 19^{+} \mathrm{CD}^{+}$subpopulation is mostly increased in $\mathrm{M}+$ patients and is responsible for the increased percentage of $\mathrm{B}$ cells observed when MS M+ patients are compared with the $\mathrm{M}$ - group.

To study intrathecal B cell activation in MS, we performed additional labeling with anti-CD20 in CSF of $9 \mathrm{M}+$ and 19
M- patients, since it has been described that disappearance of this marker is associated in mature B lymphocytes with activation and differentiation toward high-rate Ig-secreting cells (26). We found that nearly all CD5- B cells expressed CD20 in both the $M+(99.61 \% \pm 1.17 \%)$ and the $M-(99.29 \% \pm 0.41 \%)$ groups. Conversely, a fraction of $\mathrm{CD}^{+} \mathrm{B}$ cells had lost CD20 expression. This loss of CD20 was higher in the $\mathrm{M}+$ group, where only $31.45 \% \pm 5.60 \%$ of $\mathrm{CD}^{+} \mathrm{B}$ cells expressed CD20, than in the $\mathrm{M}$ - group, where $70.05 \% \pm 7.18 \%$ of $\mathrm{CD}^{+} \mathrm{B}$ cells expressed this marker $(P=0.0009$; data not shown).

Study of the specificity of the OCMBs present in a subset of MS patients. $\mathrm{CD} 19^{+} \mathrm{CD}^{+}$cells are the predominant $\mathrm{B}$ cell subpopulation present in CSF of MS M+ patients. Since these cells are characterized by the secretion of IgM antibodies that recognize nonproteic antigens, we investigated whether OCMBs were directed against myelin lipids. The presence of lipid-specific OCMBs (LS-OCMBs) was investigated in paired CSF and serum samples from 53 MS patients with OCMBs. Results are shown in Table 1.

We observed that 46 of the 53 patients (86.8\%) had LS-OCMBs. The major antigen was phosphatidylcholine (PC). Oligoclonal IgM recognizing this phospholipid was found in 37 patients. In 30 of them, PC was the only antigen recognized by the oligoclonal IgM. A representative example of this pattern is shown in Figure 3A. In the other 7 patients, an additional reactivity against other myelin lipids was also detected. A representative example (Figure 3B) shows the presence of OCMBs against PC, phosphatidylethanolamine, phosphatidylinositol, and sphingomyelin. Finally, 9 patients showed oligoclonal IgM reacting only against myelin glycolipids, sphingomyelin being the antigen most frequently recognized. The recognition of sphingomyelin by the oligoclonal IgM present in $\mathrm{CSF}$ is shown in a representative example (Figure 3C). We could not detect OCMBs against any of the myelin lipids in the 7 remaining patients. A representative example is shown in Figure 3D.

To investigate the specificity of the binding of OCMBs to the lipids, we inhibited IgM binding to the lipid-coated membranes with relevant and irrelevant antigenic preparations. A representative example (Figure 4) shows that binding of PC-specific IgM to PC-coated membranes was inhibited with this lipid but not with gangliosides, thus confirming the specificity of these antibodies.

Next, IgM specificity of $\mathrm{M}+$ patients that underwent lumbar puncture in the first stages of the disease (laboratory-supported MS) or after suffering at least 2 separate attacks (clinically definite MS; see Methods) were analyzed separately (Table 2). It was found

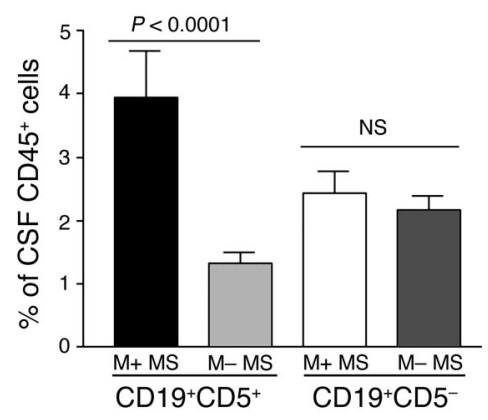

Figure 2

$B$ cell subsets in MS. The percentage of $C D 19+C D 5^{+}$cells is higher in CSF of M+ MS patients when compared with M- MS patients $(P<0.0001)$. There are no significant differences in CSF CD19+CD5cells between the 2 groups of patients. 


\section{Table 1}

Presence of LS-OCMBs in CSF of MS patients

$\begin{array}{ll}\text { No. of patients } & \text { Antigens recognized by OCMBs } \\ 30 & \text { PC } \\ 2 & \text { PC, PI } \\ 1 & \text { PC, GA } \\ 1 & \text { PC, PS } \\ 1 & \text { PC, SP } \\ 1 & \text { PC, SP, SU } \\ 1 & \text { PC, PI, PEA, SP } \\ 3 & \text { SP } \\ 2 & \text { GA } \\ 2 & \text { SU } \\ 1 & \text { SP, SU } \\ 1 & \text { SP, GA, SU } \\ 7 & \text { None }\end{array}$

PEA, phosphatidylethanolamine; PI, phosphatidylinositol; PS, phosphatidylserine; GA, gangliosides; SP, sphingomyelin; SU, sulphatides.

that all patients with a clinically definite MS at lumbar puncture had LS-OCMBs. However, only 15 of the 22 patients studied in the first stages of the disease had them.

Ten of these $\mathrm{M}+$ patients studied in the first stages of the disease had also been included in the study of CSF B cell subpopulations. The percentage of $\mathrm{CD}^{1} 9^{+} \mathrm{CD}^{+}$cells was $4.22 \% \pm 1.21 \%$ in the 7 patients with LS-OCMBs and $1.35 \% \pm 0.34 \%$ in the 3 with OCMBs that did not recognize myelin lipids. Although differences between the 2 groups were not significant $(P=0.1)$, probably due to the small number of patients studied, the percentage of $\mathrm{CD5}^{+} \mathrm{B}$ cells present in patients without LS-OCMBs resembles that of $\mathrm{M}$ - patients.

Relationship between the presence of LS-OCMBs and disease course. It has been demonstrated that the presence of OCMBs restricted to CSF is an unfavorable prognostic factor in MS $(12,14)$. We wished to evaluate whether LS-OCMBs were a more accurate prognostic marker than total OCMBs (regardless of lipid specificity). To study this hypothesis, we analyzed the evolution of $48 \mathrm{MS}$ patients that underwent lumbar puncture after the first attack of the disease. Fifteen of them showed LS-OCMBs in CSF, the other 7 showed CSF OCMBs that did not recognize myelin

\section{Figure 3}

Study of OCMB specificity. The presence of total IgM bands $(\mathrm{T})$ and of oligoclonal IgM reacting against $\mathrm{PC}$, phosphatidylethanolamine (PEA), phosphatidylinositol $(\mathrm{PI})$, phosphatidylserine (PS), sphingomyelin (SP), gangliosides (GA), sulphatides (SU), and membranes coated with Polypep (NC, negative control) were studied in paired serum (S) and CSF (C) samples from $53 \mathrm{MS}$ patients with OCMBs restricted to CSF as determined by IEF and immunodetection. Shown are representative examples of the 4 patterns that were found. (A) OCMBs against PC were detected. (B) OCMBs against PC, PEA, $\mathrm{PI}$, and SP can be observed. (C) OCMBs recognizing SP were found. (D) The OCMBs did not recognize any of the lipids studied. lipids, and the remaining 26 did not have OCMBs restricted to CSF. Clinical and immunological characteristics of these patients are summarized in Table 3.

The time elapsed between the first and the second relapse has been reported to be a prognostic marker in MS (27). We evaluated it and compared the probability of remaining free of a second relapse in this group of patients divided according to the presence or absence of OCMBs, regardless of lipid specificity (Figure 5A), or LS-OCMBs (Figure 5B). Comparisons were made by means of Kaplan-Meier tests.

Patients with OCMBs, regardless of lipid specificity, developed a second relapse earlier than patients lacking them, as we have previously described (12). Five years after disease onset, the probability of remaining free of relapses was $17.9 \%$ for patients with OCMBs and $32.5 \%$ for patients without OCMBs $(P=0.0004)$. However, differences increased dramatically when the presence or absence of LS-OCMBs was considered. All patients with LS-OCMBs suffered a second relapse 11 months after disease onset. Conversely, 36.4\% of patients lacking LS-OCMBs remained free of relapses after 60 months of follow-up $(P<0.0001)$. Thus, the presence of IgM reacting against myelin lipids strongly associates with early appearance of a second relapse in MS.

The impact of the presence of OCMBs and LS-OCMBs in the early onset of new relapses was further evaluated by multivari-

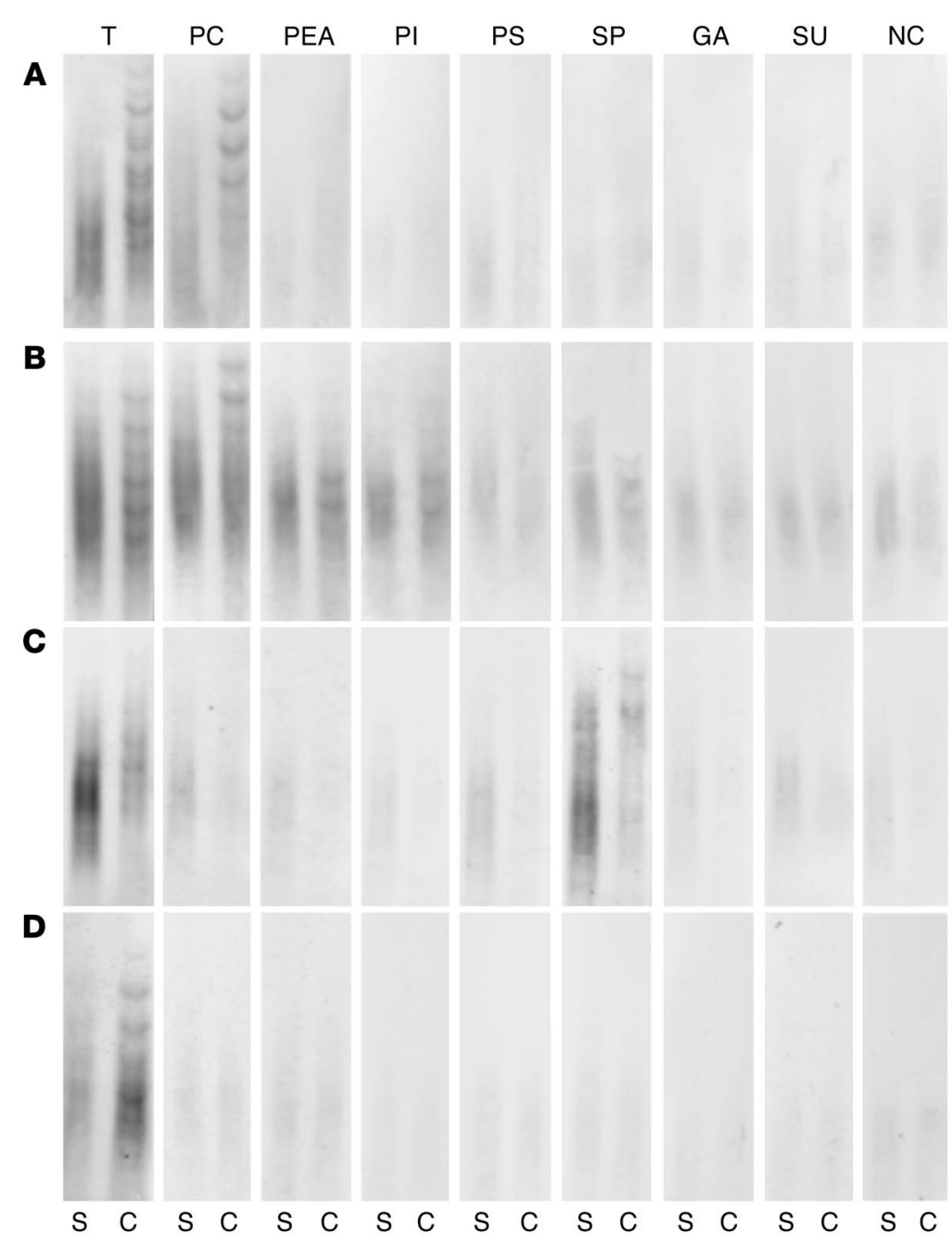




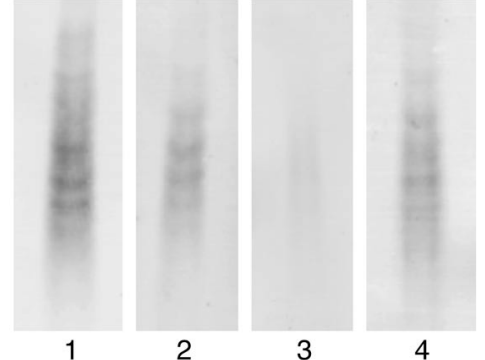

Figure 4

Inhibition of IgM binding to lipid-coated membranes. Four aliquots of CSF from a patient presenting OCMB against PC were analyzed by IEF. Proteins were transferred to: an uncoated nitrocellulose membrane (1), a PC-coated nitrocellulose membrane (2), a PC-coated nitrocellulose membrane embedded with a solution of PC that inhibits binding of specific IgM to the antigen-coated membrane (3), and a PC-coated nitrocellulose membrane embedded with a solution of gangliosides that does not produce any inhibition (4).

ate Cox regression. The presence of total OCMBs (regardless of lipid specificity) resulted in an increased risk of suffering a second relapse (hazard ratio, 3.54; confidence interval, 1.65-7.55). However, the risk was considerably higher in patients with LS-OCMBs (hazard ratio, 11.41; confidence interval, 4.26-30.58). These results confirm that LS-OCMBs are more accurate than total OCMBs in predicting the early onset of a second relapse in MS.

The relationship of the presence of LS-OCMBs with the clinical course of the disease was further explored by monitoring the number of relapses and the progression of disability during follow-up. Although IFN- $\beta$ treatment is known to be effective in decreasing the number of relapses in MS patients (28-30) and could introduce bias in our study, for ethical reasons treatment was offered to all patients who had suffered at least 2 relapses 6 months after disease onset. The probability of remaining without treatment during follow-up was evaluated by means of a KaplanMeier test in patients with and without LS-OCMBs. Results are shown in Figure 6. The probability of remaining without treatment was $0 \%$ after 24 months of follow-up for patients with LS-OCMBs. Conversely, at the end of the study, it was still $55.7 \%$ for patients without LS-OCMBs $(P<0.0001)$.

The number of relapses during follow-up was evaluated in both groups. Although most patients with LS-OCMBs began IFN- $\beta$ treatment earlier than those without LS-OCMBs, the former suffered more relapses $2.7(2.7 \pm 0.7)$ than the latter $(0.8 \pm 0.2)$ $(P=0.0004)$. Results are shown Figure 7A.

The neurological disability experienced by the patients during the study was also evaluated. Although it has been reported that increase in the expanded standard disability status scale (EDSS; see Methods) score is generally moderate in the first few following the disease onset, after follow-up, the score was $1.68 \pm 0.36$ for patients with LS-OCMBs and $0.85 \pm 0.16$ for those lacking LS-OCMBs $(P=0.02)$ (Figure 7B).

As we have demonstrated, the presence of LS-OCMBs not only associates with a higher number of relapses but also with greater disability in MS, even at the first stages of the disease.

\section{Discussion}

Increasing evidence suggests that IgM antibodies play an important role in the evolution of MS in a group of patients. The pres- ence of CSF-restricted OCMBs seems to be characteristic of these MS patients throughout the course of the disease $(13,14)$ and is associated with a more aggressive disease course $(11,12,14)$. Sustained IgM responses like this are usually produced by the long-lived $\mathrm{CD}^{+}$subset of B lymphocytes (19), which is increased in CSF of patients with aggressive forms of MS (21). These B cells are characterized by the secretion of IgM antibodies that recognize phylogenetically conserved structures (natural antibodies) (19). B cell subpopulations of MS patients divided according to the presence or absence of OCMBs were firstly studied to investigate the nature of the $\mathrm{B}$ cells responsible for the secretion of oligoclonal $\operatorname{IgM}$ bands in this disease. A very significant increase in $\mathrm{CD}_{19}{ }^{+} \mathrm{CD}^{+}$lymphocytes was found in CSF of MS patients with OCMBs when compared with controls and with MS patients without intrathecal IgM synthesis. These B cells tend to be autoreactive (31) and have been implicated in the pathogenesis of autoimmunity (32). Our data suggest that OCMBs in MS can be natural antibodies secreted by $\mathrm{CD}^{+} \mathrm{B}$ cells. In addition, we have observed that most $\mathrm{CD} 19^{+} \mathrm{CD}^{+}$lymphocytes present in CSF from MS patients with intrathecal IgM synthesis have lost CD20 expression, showing a phenotype characteristic of differentiated $B$ cells capable of secreting higher amounts of immunoglobulins (26). Recently, the existence of a maturation process of B cells within CSF in MS has been postulated (33). Our results suggest that this phenomenon preferentially occurs in $\mathrm{CD}^{+} \mathrm{B}$ cells of MS patients with OCMBs restricted to CSF.

To further study the role of OCMB in MS, we analyzed the specificity of intrathecal IgM produced in this disease. A large proportion of natural IgM antibodies recognize conserved nonproteic structures such as nucleic acids, carbohydrates, and phospholipids (19). We studied the reactivity of oligoclonal IgM present in MS patients against a set of phospholipids and glycolipids, the major nonproteic components of myelin. This study included 53 MS patients with OCMBs restricted to CSF. Forty-six (86.8\%) showed reactivity against myelin lipids, with PC being the antigen most frequently recognized. These results demonstrate that in most MS patients, OCMBs are natural antibodies that recognize major myelin antigens.

Natural IgM antibodies have a pentameric structure and are strong complement activators. In MS, IgM is the only immunoglobulin whose intrathecal synthesis correlates with intrathecal complement activation (8), and the role of complement and antibodies in demyelination and axonal damage, the cause of permanent disability in this disease, has been clearly demonstrated (34). Moreover, in an experimental model, anti-myelin lipid IgM, but not IgG, antibodies induce CNS demyelination and prevent remyelination (35). These data suggest that the presence of IgM anti-

\section{Table 2}

Distribution of LS-OCMBs in patients classified according to disease duration

$\begin{array}{lccc}\text { Disease stage } & \text { No. of patients } & \text { LS-OCMB+ } & \text { LS-OCMB- } \\ \text { Clinically definite } & 31 & 31(100 \%) & 0(0 \%) \\ \text { First relapse } & 22 & 15(68.2 \%) & 7(31.8 \%)\end{array}$

LS-OCMB+, presence of LS-OCMBs; LS-OCMB-, absence of LSOCMBs; Clinically definite, patients that underwent lumbar puncture after suffering at least 2 separate attacks of MS; First relapse, patients that underwent lumbar puncture after the first attack of MS. 
Table 3

Clinical and immunological data from the patients included in the study

\begin{tabular}{|c|c|c|c|c|c|c|c|c|c|c|c|c|}
\hline Patient & Age & Sex & Evol. & F-up & 2nd rel. & No. rel. & $E$ & Alb-I & IgG-I & GB & MB & LS-OCMB \\
\hline 1 & 19 & $\mathrm{M}$ & 0.3 & 44 & 7 & 4 & 1 & 4.10 & 0.63 & + & + & + \\
\hline 2 & 21 & $\mathrm{~F}$ & 0.6 & 6 & 2 & 2 & 2 & 6.94 & 0.71 & + & + & + \\
\hline 3 & 27 & M & 8 & 12 & 10 & 1 & 1 & 5.64 & 1.35 & + & + & + \\
\hline 4 & 32 & M & 1 & 31 & 5 & 4 & 6 & 5.51 & 2.81 & + & + & + \\
\hline 5 & 19 & $M$ & 0.3 & 17 & 5 & 1 & 2 & 2.90 & 0.75 & + & + & + \\
\hline 6 & 27 & M & 5 & 12 & 5 & 1 & 0 & 6.32 & 0.61 & + & + & + \\
\hline 7 & 31 & $\mathrm{~F}$ & 0.3 & 6 & 1 & 1 & 1.5 & 2.99 & 0.73 & + & + & + \\
\hline 8 & 26 & $\mathrm{~F}$ & 0.6 & 59 & 4 & 1 & 1 & 4.13 & 0.73 & + & + & + \\
\hline 9 & 38 & $\mathrm{~F}$ & 0.5 & 15 & 11 & 3 & 1 & 2.60 & 0.48 & + & + & + \\
\hline 10 & 42 & $\mathrm{~F}$ & 1 & 31 & 2 & 1 & 1.5 & 7.51 & 0.63 & + & + & + \\
\hline 11 & 28 & $\mathrm{~F}$ & 0.1 & 17 & 5 & 4 & 2 & 5.22 & 1.17 & + & + & + \\
\hline 12 & 20 & $M$ & 0.3 & 12 & 2 & 2 & 2 & 3.52 & 0.84 & + & + & + \\
\hline 13 & 42 & $\mathrm{~F}$ & 0.5 & 6 & 3 & 1 & 1.5 & 4.60 & 1.49 & + & + & + \\
\hline 14 & 24 & $\mathrm{~F}$ & 0.25 & 56 & 6 & 12 & 2 & 2.14 & 0.56 & + & + & + \\
\hline 15 & 26 & $M$ & 0.25 & 57 & 10 & 2 & 1 & 10.01 & 0.63 & + & + & + \\
\hline 16 & 27 & M & 5 & 6 & None & 0 & 1 & 6.53 & 0.50 & + & + & - \\
\hline 17 & 34 & $M$ & 4 & 43 & None & 0 & 0 & 4.61 & 1.45 & + & + & - \\
\hline 18 & 45 & $M$ & 0.5 & 12 & 5 & 2 & 1.5 & 10.20 & 0.86 & + & + & - \\
\hline 19 & 36 & $\mathrm{~F}$ & 0.6 & 19 & None & 0 & 1.5 & 3.60 & 1.57 & + & + & - \\
\hline 20 & 21 & $\mathrm{~F}$ & 5 & 6 & 5 & 1 & 1.5 & 4.20 & 0.70 & + & + & - \\
\hline 21 & 53 & $\mathrm{~F}$ & 5 & 7 & None & 0 & 4 & 4.91 & 0.74 & + & + & - \\
\hline 22 & 30 & $M$ & 3 & 16 & None & 0 & 0 & 4.52 & 0.84 & + & + & - \\
\hline 23 & 33 & $\mathrm{~F}$ & 2 & 37 & None & 0 & 0 & 2.41 & 1.27 & + & - & ND \\
\hline 24 & 18 & $M$ & 3 & 60 & 5 & 1 & 1 & 6.59 & 0.70 & + & - & ND \\
\hline 25 & 46 & $\mathrm{~F}$ & 0.5 & 14 & None & 0 & 1 & 5.99 & 0.72 & + & - & ND \\
\hline 26 & 54 & $\mathrm{~F}$ & 3 & 12 & 12 & 1 & 1 & 6.72 & 0.59 & + & - & ND \\
\hline 27 & 33 & $\mathrm{~F}$ & 4 & 32 & 10 & 1 & 1.5 & 3.51 & 1.33 & + & - & ND \\
\hline 28 & 21 & $\mathrm{~F}$ & 0.5 & 15 & None & 0 & 0 & 4.78 & 2.29 & + & - & ND \\
\hline 29 & 14 & $M$ & 0.1 & 40 & 40 & 1 & 0 & 2.32 & 0.95 & + & - & ND \\
\hline 30 & 39 & $\mathrm{~F}$ & 6 & 22 & 4 & 3 & 0 & 3.51 & 0.52 & + & - & ND \\
\hline 31 & 49 & $M$ & 1 & 60 & None & 0 & 2 & 6.29 & 1.41 & + & - & ND \\
\hline 32 & 31 & $\mathrm{~F}$ & 0.1 & 54 & None & 0 & 0 & 4.91 & 0.90 & + & - & ND \\
\hline 33 & 26 & $\mathrm{~F}$ & 0.3 & 60 & 15 & 3 & 0 & 3.92 & 0.69 & + & - & ND \\
\hline 34 & 26 & $\mathrm{~F}$ & 0.6 & 13 & None & 0 & 0 & 6.58 & 0.85 & + & - & ND \\
\hline 35 & 23 & $\mathrm{~F}$ & 0.2 & 60 & None & 0 & 0 & 4.11 & 0.47 & + & - & ND \\
\hline 36 & 33 & $\mathrm{~F}$ & 0.1 & 20 & None & 0 & 0 & 4.59 & 0.54 & + & - & ND \\
\hline 37 & 34 & $\mathrm{~F}$ & 4 & 29 & 15 & 1 & 1 & 3.51 & 0.62 & + & - & ND \\
\hline 38 & 28 & $M$ & 0.3 & 24 & 23 & 1 & 2 & 4.42 & 0.97 & + & - & ND \\
\hline 39 & 19 & $\mathrm{~F}$ & 0.5 & 60 & 9 & 5 & 1 & 5.79 & 0.50 & + & - & ND \\
\hline 40 & 28 & $\mathrm{~F}$ & 1 & 51 & None & 0 & 0 & 2.49 & 1.35 & + & - & ND \\
\hline 41 & 19 & $\mathrm{~F}$ & 2 & 27 & 18 & 1 & 1.5 & 5.61 & 0.91 & + & - & ND \\
\hline 42 & 36 & $M$ & 1 & 19 & None & 0 & 1 & 6.48 & 1.11 & + & - & ND \\
\hline 43 & 31 & $\mathrm{~F}$ & 0.25 & 60 & 15 & 5 & 1.5 & 3.82 & 2.08 & + & - & ND \\
\hline 44 & 23 & $\mathrm{~F}$ & 0.5 & 20 & 16 & 2 & 1 & 3.48 & 1.13 & + & - & ND \\
\hline 45 & 25 & $\mathrm{~F}$ & 0.1 & 23 & 22 & 1 & 0 & 2.39 & 0.61 & + & - & ND \\
\hline 46 & 33 & $\mathrm{~F}$ & 0.5 & 57 & None & 0 & 1.5 & 6.22 & 0.92 & + & - & ND \\
\hline 47 & 37 & $\mathrm{~F}$ & 6 & 13 & None & 0 & 1 & 3.21 & 0.8 & + & - & ND \\
\hline 48 & 61 & $\mathrm{~F}$ & 0.5 & 12 & None & 0 & 1 & 7.83 & 0.89 & + & - & ND \\
\hline
\end{tabular}

F, female; M male; Evol., time elapsed since disease onset (mo); F-up, time of follow-up (mo); 2nd rel., number of months elapsed until the second relapse, if any; No. rel., number of relapses suffered during the follow-up; E, EDSS score at the end of the study; Alb-I, albumin index; IgG-I, IgG index; GB, presence of oligoclonal IgG bands restricted to CSF; MB, presence of OCMBs restricted to CSF; LS-OCMB, presence of LS-OCMB; ND, not determined.

myelin lipids in CSF can be important in the physiopathology of MS. To investigate this, we studied the relationship of these antibodies with disease course in a group of patients in whom the presence of OCMBs was studied at disease onset. In this group were 15 patients with LS-OCMBs, 7 patients with OCMBs that did not recognize myelin lipids, and 26 patients lacking IgM bands. First we evaluated the probability of remaining free of relapses.
We found that patients with LS-OCMBs developed a second relapse earlier than those without LS-OCMBs. Differences were more significant than those obtained when comparing patients with and without OCMBs, regardless of lipid specificity, and indicate a strong correlation between the presence of anti-lipid IgM antibodies and an aggressive disease course in MS. These data were confirmed by evaluating the number of relapses and the dis- 
A

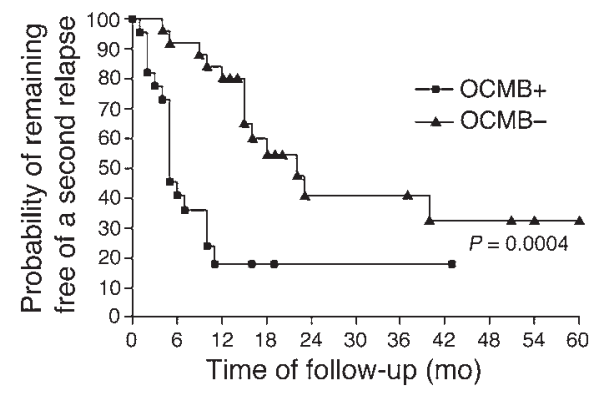

B

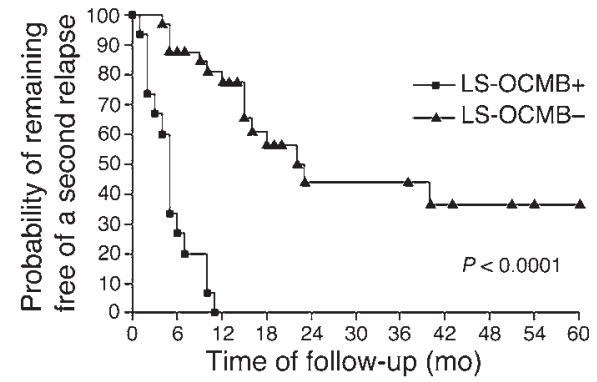

Figure 5

Probability of remaining free of a second relapse, which was evaluated by a Kaplan-Meier test in $48 \mathrm{MS}$ patients that had undergone lumbar puncture after the first attack of the disease. First, the disease course in the 22 patients with OCMBs was compared with that of the 26 patients without them (A). Secondly, the disease course in the 15 patients with LS-OCMBs (LS-OCMB+) was compared with that of the group formed by the 26 patients without OCMBs plus the 7 patients with OCMBs that did not recognize myelin lipids (LS-OCMB-) (B).

ability. Patients with LS-OCMBs suffered more relapses during follow-up and had higher disability scores at the end of the study than those without LS-OCMBs.

It can be concluded that the presence of LS-OCMBs is a more accurate prognostic factor in MS than that of total OCMBs. This can be explained by the presence of OCMBs that do not recognize myelin lipids, which may represent a transient primary response not associated with an aggressive disease course.

Our results strongly suggest that local synthesis of LS-OCMBs plays an important role in MS physiopathology. These antibodies may exacerbate the disease not only by inducing complementdependent demyelination, but can also by enhancing myelin phagocytosis by macrophages and microglia via $\mathrm{Fc}$ and complement receptors. This can improve presentation of proteic myelin antigens in the context of MHC class II molecules and of lipid myelin antigens in the context of CD1 molecules. This hypothesis is in line with previous findings describing that both CD1 and MHC class II molecules are upregulated in APCs present in active MS lesions $(36,37)$. By means of these mechanisms, LS-OCMBs can collaborate to expand the inflammatory reaction that takes place in MS, contributing to lesion development.

\section{Methods}

Samples. CSF and peripheral blood samples from MS patients and from patients with nonspecific headaches (control group) were studied. They were obtained for diagnostic purposes after we received informed consent.

$\mathrm{CSF}$ and sera samples were aliquoted and stored at $-40^{\circ} \mathrm{C}$. For the analysis of B cell subpopulations, fresh CSF samples $(6-8 \mathrm{ml})$ were centrifuged at $500 \mathrm{~g}$ for 15 minutes and the cellular pellet processed, as described below.
Patients. This study was approved by the ethical committee of Hospital Ramón y Cajal. MS patients were diagnosed according to Poser's criteria (38). Patients fulfilled the criteria for laboratory-supported MS (a first relapse, evidence of 2 lesions and oligoclonal IgG bands) or clinically definite MS (at least 2 separate attacks). During the diagnosis and followup, at least 1 MRI study was performed on each patient. All the patients showed 2 or more lesions of the brain, suggestive of demyelination, that were at least $3 \mathrm{~mm}$ in diameter on $\mathrm{T}_{2}$-weighted MRI scans, with at least 1 of them being periventricular or ovoid. All patients showed oligoclonal IgG bands restricted to CSF.

The main purposes of this study were the identification of the B cell subpopulation responsible for intrathecal $\mathrm{IgM}$ secretion in MS, the analysis of the specificity of OCMBs presents in MS patients, and determining whether there is a correlation of specific bands with the aggressiveness of the disease. Eighty-one MS patients and 21 controls were studied to analyze CSF B cell subsets. The MS group included 29 patients with CSF OCMBs and 52 without them. Fifty-three MS patients with CSF OCMBs were studied to analyze the specificity of IgM bands. In 26 of these patients, B cell study had also been performed. Forty-eight patients with laboratory-supported MS were followed up to study the relationship between IgM specificity and disease evolution in MS. Twenty-two had oligoclonal IgM restricted to CSF. All of them had been included in the study of IgM specificity. Of these, 15 had LS-OCMBs and 7 had OCMBs that did not recognize myelin lipids. The other 26 patients had no OCMBs. B cell studies had also been performed on samples from 19 patients from this group.

Patients were followed up for a variable period of time ranging from 6 to 60 months (mean $\pm \mathrm{SE}, 31.17 \pm 3.54$ months). The mean time of follow-up was $25.4 \pm 5.0$ months for patients with LS-OCMBs and $30.4 \pm 3.4$ months for patients without LS-OCMBs. Differences in the time of followup between both groups were not significant.

Patient follow-up. A neurologist examined patients at lumbar puncture and every 6 months until the end of the study, in a blinded manner, with additional neurological assessment in case of relapses. Relapses were defined according to Poser et al. (38) as a worsening of neurological impairment or an appearance of a new symptom or abnormality attributable to MS lasting at least 24 hours and preceded by stability of at least 1 month. Disability was measured using the EDSS, according to which scores range from 0 (no disability) to 10 (death). EDSS scores were assessed at each visit. When the procedure was performed during a relapse, a new EDSS score was recorded a month later, when the neurological situation had stabilized. In the first visit, oligoclonal IgG bands, OCMBs, LS-OCMBs, and EDSS score were recorded. During the follow-up, the time to a second relapse, the number of relapses, and the variations in the EDSS score were studied.

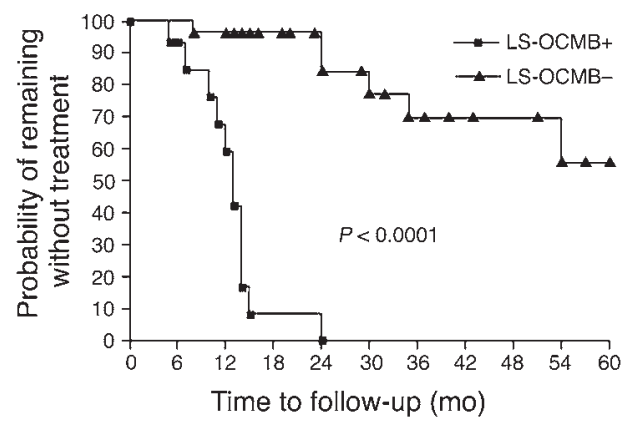

\section{Figure 6}

Probability of remaining free of IFN- $\beta$ treatment during follow-up. It was lower in the 15 patients with CSF-restricted LS-OCMBs (LS-OCMB+) than in the 33 patients without LS-OCMBs (LS-OCMB-) $(P<0.0001)$. 

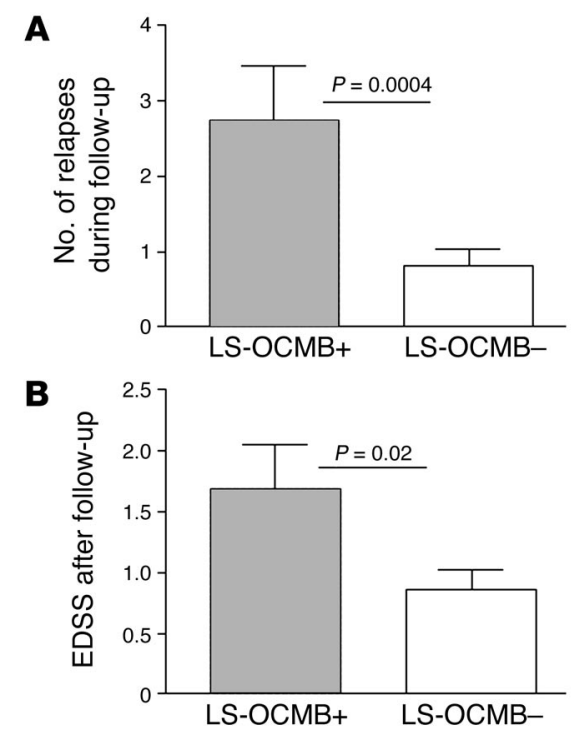

Treatment during follow-up. Patients were treated for relapses intravenously with $1 \mathrm{~g} / \mathrm{d}$ methylprednisolone for 5 days with an oral prednisone taper. Treatment with IFN- $\beta-1 b$ (Betaferon; Schering España) and IFN- $\beta-1 \mathrm{a}$ (Avonex [Biogen Idec] and Rebif [Serono España]) is approved in Spain for patients with relapsing-remitting MS for at least 6 months and with 2 relapses or more in the previous 3 years. IFN- $\beta$ treatment was offered to all patients included in this study who fulfilled these criteria. Eight patients were treated with Rebif, 5 with Avonex, and 5 with Betaferon.

Oligoclonal band detection. IgG and IgM oligoclonal bands were analyzed by isoelectric focusing (IEF) and immunoblotting, as previously described $(10,39)$.

IgM quantification. Serum IgM was quantified by nephelometry in a Dade Behring nephelometer. CSF IgM was quantified by an ELISA sandwich assay involving the recognition of IgM by a purified mouse monoclonal anti-human IgM (Tago) linked to the ELISA plate and a goat anti-human IgM labeled with peroxidase (Jackson ImmunoResearch Laboratories Inc.). A serum sample with a known amount of IgM was used as standard.

Labeling of cells and flow cytometry. The following $\mathrm{mAbs}$ were used: control IgG1-FITC, IgG1-PE, IgG1-peridinin-chlorophyll-protein-Cy-Chrome5.5 (IgG1-PerCP-Cy5.5), CD20-FITC, CD19-PerCP-Cy5.5, and CD45-APC were from BD; CD5-PE was from Beckman Coulter. CSF cells were washed, and 4 labeling experiments were performed with optimal concentrations of conjugated mAbs for 30 minutes at $4{ }^{\circ} \mathrm{C}$. The cells were washed twice and analyzed on a standard FACSCalibur instrument (BD). Cell scatter gating was set to include small (resting lymphocytes) and large (activated lymphocytes, plasma cells, and monocytes) cells and to exclude apoptotic cells. A minimum of 500 events were collected for analysis of antigen staining. The cursor was set so that less than $1 \%$ of the cells in each sample stained positively with the negative control mAbs. The percentage of cells that stained positively was recorded for each sample.

Detection of LS-OCMBs. Stock solutions of purified PC, phosphatidylethanolamine, phosphatidylinositol, phosphatidylserine, sphingomyelin, gangliosides, and sulphatides (Sigma-Aldrich) were prepared at $10 \mathrm{mg} / \mathrm{ml}$ in 2:1 chloroform/methanol. Lipid micelles were prepared by diluting each stock solution in saline at a final lipid concentration of $20 \mu \mathrm{g} / \mathrm{ml}$ and vortexing.

Seven nitrocellulose membranes (Amersham Biosciences) were incubated overnight at $4{ }^{\circ} \mathrm{C}$ in a shaker, each one with $20 \mathrm{ml}$ of 1 of the lipid micelle solutions previously prepared; an eighth was incubated in $20 \mathrm{ml}$ saline, and

\section{Figure 7}

Evaluation of disease course. The number of relapses during followup (A) and the neurological disability at the end of the study (B) were monitored in patients with and without LS-OCMBs. Comparisons were made by a Mann-Whitney $U$ test. Patients with LS-OCMBs had more relapses $(P=0.0004)$ and became more disabled $(P=0.02)$ than those without LS-OCMBs.

a ninth was not treated. After incubation, the membranes incubated either with lipids or with saline were washed in 3 changes of $1 \%$ Polypep (SigmaAldrich) in saline for 1 hour and blocked 18 hours at $4{ }^{\circ} \mathrm{C}$ in $20 \mathrm{ml}$ of $3 \%$ Polypep in saline. Before use, they were rinsed in saline.

To investigate whether oligoclonal IgM recognizes any of the myelin lipids, we applied 9 sets of a serum and a paired CSF sample from each MS patient with OCMBs to the IEF gel and subjected them to IEF in order to separate IgM, as previously described (10). After IEF, proteins were transferred to the 9 nitrocellulose membranes prepared previously. The transfer was carried out by placing the membranes on the gel surface, covering them with 25 sheets of dry filter paper, and placing this under a 2 -kg weight for 20 minutes. The presence of OCMBs linked to the membranes was investigated by immunoblotting with a phosphatase alkaline anti-human IgM antibody (Jackson ImmunoResearch Laboratories Inc.) as previously described (10).

Inbibition of IgM binding to lipid-coated membranes. Eight nitrocellulose membranes were incubated with PC micelles and blocked with Polypep as described above. Nine sets of a CSF sample from a patient with OCMBs recognizing PC were analyzed by IEF, as described above. After IEF, a sample was transferred to an uncoated PVDF membrane, a second one to a PC-coated membrane, and the remaining ones to PC-coated membranes that immediately prior to transfer had been embedded in $50 \mathrm{mg} / \mathrm{ml}$ solutions of PC, phosphatidylethanolamine, phosphatidylinositol, phosphatidylserine, sphingomyelin, gangliosides, and sulphatides, respectively, to determine whether these lipid solutions inhibit IgM binding to the lipid-coated membrane. Lipid solutions were prepared in saline serum containing 3\% Polypep. The transfer was carried out as described above. The membranes were then washed 25 times with tap water and an additional 25 times with saline serum containing $3 \%$ Polypep. Finally, the presence of OCMBs linked to the membranes was investigated by immunoblotting, as described above.

Statistics. We used Mann-Whitney $U$ test for comparisons between groups, Kaplan-Meier analysis for survival curve comparisons, and multivariate Cox regression for hazard ratio calculation. $P$ values of less than 0.025 were considered statistically significant. Mann-Whitney $U$ and Kaplan Meier tests were performed with GraphPad Prism statistical package (version 3.0). Cox regression was calculated with SPSS statistical package (version 10).

\section{Acknowledgments}

This work was supported by grant PI020043 from the Fondo de Investigaciones Sanitarias (Spain). M.C. Sádaba was supported by a fellowship grant from the LAIR foundation. We acknowledge A. Muriel for his valuable help with statistical tests.

Received for publication July 27, 2004, and accepted in revised form October 19, 2004.

Address correspondence to: Luisa María Villar, Servicio de Inmunología, Hospital Ramón y Cajal, Carretera de Colmenar km. 9.100, Madrid 28034, Spain. Phone: 34-913368795; Fax: 34-913368809; E-mail: lvillar.hrc@salud.madrid.org. 
1. Genain, C.P., Canella, B., Hauser, S.L., and Raine, C.S. 1999. Identification of the autoantibodies associated with myelin damage in MS. Nat. Med. 5:153-154.

2. Archelos, J.J., Storch, M.K., and Hartung, H.P. 2000. The role of B cells and antibodies in MS Ann. Neurol. 47:694-706.

3. Derfuss, T., et al. 2001. Intrathecal antibody production against Chlamydia pneumoniae in MS is part of a polyspecific immune response. Brain 124:1325-1335.

4. Cross, A.H., Trotter, J.L., and Lions, J.A. 2001. B cells and antibodies in CNS demyelinating disease. J. Neuroimmunol. 112:1-14.

5. Lucchinetti, C., et al. 2000. Heterogeneity of MS lesions: implications for the pathogenesis of demyelination. Ann. Neurol. 47:707-717.

6. Gay, F.W., Drye, T.J., Dick, G.W., and Esiri, M. 1997. The application of multifactorial cluster analysis in the staging of plaques in early MS. Brain. 120:1461-1483.

7. Barnett, M.H., and Prineas, J.W. 2004. Relapsing and remitting MS: pathology of the newly forming lesion. Ann. Neurol. 55:458-468.

8. Sellebjerg, F., Christiansen, M., and Garred, P. 1998. MBP, anti-MBP and anti-PLP antibodies, and intrathecal complement activation in MS. Mult. Scler. 4:127-131.

9. Frequin, S.T.F.M., Barkhof, F., Lamers, K.J.B., Hommes, O.R., and Borm, G.F. 1992. CSF myelin basic protein, IgG and IgM levels in $101 \mathrm{MS}$ patients before and after treatment with high-dose intravenous methylprednisolone. Acta Neurol. Scand. 86:291-297.

10. Villar, L.M., et al. 2001. A sensitive and reproducible method for the detection of oligoclonal IgM bands. J. Immunol. Methods. 258:151-155.

11. Villar, L.M., et al. 2002. Intrathecal IgM synthesis in neurological diseases. Relationship with disability in MS. Neurology. 58:824-826.

12. Villar, L.M., et al. 2002. Intrathecal IgM synthesis predicts the onset of new relapses and a worse disease course in MS. Neurology. 59:555-559.

13. Walsh, M.J., and Tourtellotte, W.W. 1986. Temporal invariance and clonal uniformity of brain and cerebrospinal IgG, IgA and IgM in MS. J. Exp. Med. 163:41-53.

14. Villar, L.M., et al. 2003. Intrathecal IgM synthesis is a prognostic factor in MS. Ann. Neurol. 53:222-226.

15. Piddlesden, S.J.H., Lassmann, H., Zimprich, B.P., Morgan, B.P., and Linington, C. 1993. The demyelinating potential of antibodies to myelin oligodendrocyte glycoprotein is related to their ability to fix complement. Am. J. Pathol. 143:555-564.

16. Mead, R.J., Singhrao, S.K., Neal, J.W., Lassmann, H., and Morgan, B.P. 2002. The membrane attack complex of complement causes severe demyelination associated with acute axonal injury. J. Immunol. 168:458-465.

17. Lucchinetti, C.F., et al. 2002. A role for humoral mechanisms in the pathogenesis of Devic's neuromyelitis optica. Brain. 125:1450-1461.

18. Berland, R., and Wortis, H.H. 2002. Origins and functions of B-1 cells with notes on the role of CD5. Annu. Rev. Immunol. 20:253-300.

19. Boes, M. 2000. Role of natural and immune IgM antibodies in immune responses. Mol. Immunol. 37:1141-1149.

20. Mix, E., et al. 1990. B cells expressing CD5 are increased in cerebrospinal fluid of patients with MS. Clin. Exp. Immunol. 79:21-27.

21. Bongioanni, P. 1996. Lymphocyte subsets in MS. A study with two-colour fluorescence analysis. J. Neurol. Sci. 139:71-77.

22. O'Brien, J.S., and Sampson, L.E. 1965. Lipid composition of the normal human brain: gray matter, white matter, and myelin. J. Lipid Res. 6:537-544.

23. Deber, C.M., and Reynolds, S.J. 1991. Central nervous system myelin: structure, function and pathology. Clin. Biochem. 24:113-134.

24. Dabby, R., Weimer, L.H., Hays, A.P., Olarte, M., and Latov, N. 2000. Antisulfatide antibodies in neuropathy. Neurology. 54:1448-1452.

25. Alaedini, A., Sander, H.W., Hays, A.P., and Latov, N. 2003. Antiganglioside antibodies in multifocal acquired sensory and motor neuropathy. Arch. Neurol. 60:42-46.

26. Jensen, G.S., Poppema, S., Mant, M.J., and Pilarski, L.M. 1989. Transition in CD45 isoform expression during differentiation of normal and abnormal B cells. Int. Immunol. 1:229-236.

27. Confavreux, C., Vukusic, S., and Adeleine, P. 2003 Early clinical predictors and progression of irreversible disability in MS: an amnesic process. Brain.
126:770-782.

28. The IFNB Multiple Sclerosis Study Group. 1993. Interferon beta-1b is effective in relapsing-remitting MS, I: clinical results of a multicenter, randomized, double-blind, placebo-controlled trial. Neurology. 43:655-661.

29. Jacobs, L.D., et al. 1996. Intramuscular interferon beta-1a for disease progression in relapsing MS: the Multiple Sclerosis Collaborative Research Group (MSCRG). Ann. Neurol. 39:285-294.

30. PRISMS (Prevention of Relapses and Disability by Interferon $\beta$-1A Subcutaneously in Multiple Sclerosis) Study Group. 1998. Randomised double blind, placebo controlled study of interferon beta- $1 \mathrm{a}$ in relapsing-remitting MS. Lancet. 352:1498-1504.

31. Berland, R., and Wortis, H.H. 2002. Origins and functions of B-1 cells with notes on the role of CD5. Annu. Rev. Immunol. 20:253-300.

32. Rothstein, T. 2002. Cutting edge commentary: two B-1 or not to be one. J. Immunol. 168:4257-4261.

33. Corcione, A., et al. 2004. Recapitulation of B cell differentiation in the central nervous system of patients with MS. Proc. Natl. Acad. Sci. U. S. A. 101:11064-11069.

34. Mead, R.J., Singhrao, S.K., Neal, J.W., Lassmann, H., and Morgan B.P. 2002. The membrane attack complex of complement causes severe demyelination associated with acute axonal injury. J. Immunol. 168:458-465.

35. Rosenbluth, J., Schiff, R., Liang, W.L., and Dou, W. 2003. Antibody-mediated CNS demyelination II. Focal spinal cord lesions induced by implantation of an IgM antisulfatide-secreting hybridoma. J. Neurocytol. 32:265-276.

36. Battistini, L., Fischer, F.R., Raine, C.S., and Brosnan, C.F. 1996. CD1b is expressed in multiple sclerosis lesions. J. Neuroimunol. 67:145-151.

37. Trapp, B.D., Bö, L., Mörk, S., and Chang, A. 1999. Pathogenesis of tissue injury in MS lesions. J. Neuroimmunol. 98:49-56.

38. Poser, C.M., et al. 1983. New diagnostic criteria for MS: guidelines for research protocols. Ann. Neurol. 13:227-231.

39. Sádaba, M.C., et al. 2004. An ultrasensitive method for the detection of oligoclonal IgG bands. J. Immunol. Meth. 284:141-145. 